Highlights

The Right Lateral Prefrontal Cortex Impacts Control Perception as a Function of Probabilistic Stimulus Processing

André Forster,Johannes Rodrigues,Philipp Ziebell,Joseph L Sanguinetti,John JB Allen,Johannes Hewig

- The right lateral prefrontal cortex processes a stimulus' likelihood of occurrence

- EEG alpha power in the IPFC contributes to the emergence of control perception

- Low intensity ultrasonic neuromodulation is capable of modulating the right lateral prefrontal cortex, its processing properties and other structures that are functionally related to it 


\title{
The Right Lateral Prefrontal Cortex Impacts Control Perception as a Function of Probabilistic Stimulus Processing
}

\author{
André Forster $^{a, *}$, Johannes Rodrigues ${ }^{a}$, Philipp Ziebell ${ }^{a}$, Joseph L Sanguinetti ${ }^{b}$, John JB Allen ${ }^{b}$ and \\ Johannes Hewig ${ }^{a}$ \\ a Julius-Maximilians-University of Würzburg, Marcusstraße 9-11, Würzburg, 97070, Bavaria, Germany \\ ${ }^{b}$ University of Arizona, , Tucson, , Arizona, United States of America
}

\section{ARTICLE INFO}

\section{Keywords:}

Control Perception

Transcranial Ultrasound

IPFC

Mid-Frontal Theta

\begin{abstract}
A B S T R A C T
Being able to control inner and environmental states is a basic need of living creatures. The perception of such control is based on the perceived ratio of outcome probabilities given the presence and the absence of behavior. If behavior increases the perceived probability of a given outcome, actionoutcome contingency is met, and control perception (CP) may emerge. Nonetheless, regarding this model, not much is known about how the brain processes CP from this information. This study uses low-intensity transcranial focused ultrasound neuromodulation in a randomized-controlled doubleblind cross-over design to investigate the impact of the right lateral prefrontal cortex on this process. Forty healthy participants visited the laboratory twice (once in a sham, once in a neuromodulation condition) and rated their control perception regarding a classical control illusion task. EEG alpha and theta power density were analyzed in a hierarchical single trial-based mixed modeling approach. Results indicate that the lateral PFC modulates $\mathrm{CP}$ by providing stochastic information about a given situation by reacting with increased alpha responses during phases of low controllability (in which the ratio of probabilities is one). Furthermore, neuromodulation of the right IPFC was found to modulate mid-frontal theta by altering its relationship with self-reported effort and worrying. These data provide evidence for right lateral PFC-mediated probabilistic stimulus processing during the emergence of CP and DLPFC-driven modulation of cingulate functioning as indicated by midline theta changes.
\end{abstract}

\section{Introduction}

Having a sense of control over both inner and environmental states is a basic need of living creatures, including humans. In order to achieve such, as there usually is no complete data about a given situation present, an organism needs to form subjective rather than objective conclusions on whether alterations of states are contingent responses to its own action ([62]; [4]; [1]; [57]). As a result, individual differences in control-beliefs may stem from interactions of self-perceived agentic behavior and the evaluation of contingency of its consequences ([54]; [11]; [73]). As these individual differences in control expectation are implicated in a variety of psychiatric disorders such as depression ([32]; [4]) and may also pose as a trait to predetermine future episodes of such ([6]; [8]), being able to induce a greater sense of control, is a key aspect of both, preventive and curative interventions ([76]; [16]; [48]; [7]; [31]; [36]).

One method to alter control perception in individuals who are otherwise resistant to interventions is non-invasive neuromodulation ([13]; [22]; [75]). In the current study, lowintensity transcranial focused ultrasound neuromodulation (litFUS) was applied to increase the sense of control over an unpredictable rewarding stimulus by targeting the right inferior gyrus (rIFG) of the lateral prefrontal cortex (IPFC). litFUS is a novel method to precisely inhibit or excite neural ensembles even in subcortical structures. Its effect is investigated with regard to self-report measures as well as EEG measures within individual alpha and theta frequency bands.

\subsection{Neural Bases of Control Perception.}

Especially in the context of the learned helplessness theory, control perception (CP) was subject to extensive research in regards to its protective properties against stress and disease (e.g., [17]; [4]). In the theory's beginnings, to stimulate further research on the topic of learned helplessness/hopelessness, Maier and Seligman ([52],[53]) provided an influential definition for the emergence of subjective CP. The authors hypothesize control perception to ground on the estimated ratio between two perceived probabilities: First, the probability of a specified outcome following some kind of action by the subject. Second, the probability of this outcome in the absence of such action. As a result, if the subject's behavior changes the probability of an outcome in comparison to its probability in the absence of this behavior, action-outcome contingency is perceived. In turn, this experience of contingency paves the way to the overall sense of control over the outcome.

In summary, this model implies control perception to be a function of conditional probabilistic learning that then guides behavior-outcome contingency detection on which control perception builds.

The neural basis of contingency perception has been extensively researched throughout the past decades and produced several models including several regions of interest. In this regard, especially the anterior cingulate cortex (ACC) has been repeatedly shown to modulate adaptive behavior based on current action-outcome associations (for a review of studies see [37]). For instance, ACC lesions in monkeys critically impaired their ability to sustain rewarded behavior, which the authors attribute to failure to utilize information 
of the recent reinforcement history to compute the potential value of behavioral decisions ([45], also see [42]). Interestingly, this was true for both, error- and reward-driven learning processes. In line with this, Cavanagh and Shackman ([21]) state in their meta-analytic review that ACC activity reflects both, prediction errors (with implications in anxiety and conflict processing) and adaptive behavior by signaling the need for control, enhancing the attention to relevant stimuli and initiating cognitive control mechanisms in general.

On that note, one integrative model postulated by Holroyd and Umemoto ([37]) depicts several modules to interact on a hierarchical basis during goal-directed behavior and its online evaluation. Here, the ACC determines which task or objective to pursue as a function of its overall estimated value weighted against the necessary effort. It thus increases activity to regulate effortful behavior to the point when the effort is not worth the reward anymore.

\subsubsection{Lateral Prefrontal involvement in Expectancy and Control Perception}

In addition to the ACC, according to Holroyd's and Umemoto's model, the IPFC and the dorsal striatum are the main actors of the brain, connecting internal signal processing with behavioral outputs. Furthermore, this network is thought to be effort-averse, avoiding low reward/high effort tasks and behavior; i.e. for instance, tasks in which no meaningful behavior can be found to achieve a set goal. In this regard, especially the expectation to be able to get a reward seems to be closely connected to IPFC activity, highlighting its role in the processing of probabilistic information, which it then uses to modulate ACC activity. Along this line, several studies allude to the idea of IPFC orchestrated ACC activation during changes in current task rules or -demands that depend on the overall estimation that ACC-related cognitive control will be necessary for the near future (e.g., [74]; [67]; [47]).

Correspondingly, the IPFC has already been described several times as an area of interest in the context of contingencylearning and associative learning (e.g. [30]; [71]; [2]; [58]) as well as CP ([77]; [51]). For instance, Lorenz et al. ([51]) show that activity of the right inferior frontal gyrus (rIFG) of the IPFC in combination with the ventral striatum (VS) may correlate to the illusion of control. In line with this, Wiech et al. ([77]) show that activity of the right IPFC was connected to the general belief to be in control of one's life, which was connected to less pain and anxiety-related rating.

Interestingly, especially the right hemispheric IPFC may serve a unique function as evidence suggests it to be sensitive to probabilistic learning and "model building" ([55]; [65]; [27]; [68]; [41]; [29]), including probabilistic processing of associative relationships ([30]).

Even though some evidence also suggests the left IPFC to serve similar functions ([79]; [35]), findings in favor of right hemispheric dependence in stochastic information processing prevails. Thus, in summary, the IPFC, and especially the right IPFC, may either provide or at least process information about the above-mentioned estimation of probability ratios that ultimately build the basis for overall control perception as suggested by Maier and Seligman.

Taken together, given a situation of uncertainty, the ACC would be predicted to respond by increased activity reflecting the need for control, facilitating effort despite unclear probabilities of reward. Hence, it may pose as a neural basis of behavioral adaptation ([14]; [19]; [20]; [21]). However, at some point, its activity may cease due to devaluation of the anticipated outcome in the context of the escalating effort to maintain behavioral activity. According to the model of Holroyd and Umemoto, this effect may be mediated by the effort averse IPFC, which may do so, based on the processing of stochastic information, which is well in line with work highlighting DLPFC activity to depend on the expectation of events that need cognitive control to achieve a goal.

\subsection{Neuromodulation of the personal sense of control.}

One method to intervene directly in the above-mentioned neural systems is non-invasive neuromodulation. However, to our knowledge, only one study has directly investigated changes in the perception of control in response to such treatment. Borckardt et al. ([13]) increased the activity of the left IPFC via transcranial magnetic stimulation (TMS) in a task that required participants to exercise control via fast button presses over the onset of heat-induced pain. Even though unbeknownst to the participants, the reaction time of their response did not influence the pain-inducing mechanism, TMS-receiving participants reported significantly less perception of control than the sham group did. The authors concluded that the IPFC plays a role in CPmediated analgesic placebo effects. Interestingly, this result in combination with the findings by both, Wiech et al. ([77]) and Lorenz et al., ([51]) indicates an asymmetrical contribution of IPFC activity to the perception of control, as left-hemispheric activity decreases, while right-hemispheric activity increases the illusion of control.

Taken together, several target regions for neuromodulation of control perception emerge, including the ACC and the IPFC. However, in general, targeting any of these regions faces substantial issues before the background of spatial resolution and depth of modulation.

\subsubsection{Low-Intensity Focused Ultrasonic Neuromodulation}

One novel method, low-intensitity transcranial focused ultrasononic neuromodulation (litFUS) is capable to reach even subcortical regions. litFUS is a novel method to excite and inhibit neural tissue via ultrasound with a high spatial resolution and the capability to reach structures as deep as the brain stem ([49]; [70]). Several studies throughout the recent years have shown its potency in modulating neural tissue in humans ([66]; [63]; [34]) and animals ([46]; [80]), giving rise to several theories of functioning mechanisms (e.g. [72]; [61]; [5]). 
In the current study, litFUS was used to target (inhibit) the right inferior frontal gyrus, which we anticipate to be reflected by increased alpha $(8-13 \mathrm{~Hz})$ activity at the F8 electrode position in the EEG. This hypothesis builds on findings that alpha oscillations (partly) rely on T-type $\mathrm{Ca}^{2+}$ channels that pose as one mechanism of thalamus-driven synchronization (see [10]n for a short review). These channels inherently fire at a rate of approximately $10 \mathrm{~Hz}$, inducing alpha synchronization via thalamic relays ([12]; [15]; [39]; [38]). Following the neuronal intramembrane cavitation excitation model (NICE-model) of channel-specific effects of ultrasonic neuromodulation ([61]), neurons expressing this type of channel play a crucial role in delivering inhibitory effects by the (generally exciting) ultrasound waves since excited inhibitory interneurons of this type may decrease net network-level activity.

\subsection{The present study.}

Though a fair amount of studies have investigated expectation and cognitive control-related neural correlates in the IPFC, only a few studies investigated its role in control perception. Therefore, the present study seeks to tackle this research gap by using litFUS to investigate the causal relationship between right IPFC activity and $\mathrm{CP}$.

As stated above, this relationship is thought to be grounded on the processing of probabilities. Thus, a hierarchical design of analyses was chosen to relate single-trial-based reactions to probabilistic contexts to higher-order self-rate measures of $\mathrm{CP}$. As a result, the current study uses a hierarchical mixed modeling approach to assess its role in a control illusion task. On a single trial level, sequence effects of the experience with the stimulus were analyzed while on a higher level within-subject effects could be investigated.

Furthermore, mid-frontal theta (MFT) was analyzed. MFT is thought to reflect ACC activity. It is investigated as it is (a) thought to interact with the right IPFC's information processing of conditional probabilities ([19]) and (b) reflects an important area involved in contingency processing itself. Furthermore, it is thought to reflect effortful control and conflict processing considering goal-directed behavior.

In sum, an EEG was recorded during a control illusion task, asking participants to gain as much control as possible over an (unbeknownst to them) uncontrollable, rewarding stimulus. Additionally, each participant completed this task twice, once in a sham, and once in a neuromodulation condition making this a randomized controlled double-blind cross-over study design. Dependent measures were EEGbased MFT frequency-band-density-power (to inspect ACC activity) and alpha frequency-band-density-power at right IPFC regions as well as psychometric self-report measures.

\subsubsection{Hypotheses}

\subsubsection{The role of the right IPFC in control perception.}

Since the right $1 \mathrm{PFC}$ has been reported to "model the world" in a stochastic fashion, we hypothesize that the neuromodulation interferes with its processing of reinforcer presentation rates. Thus, the alpha power density at the F8 electrode position (10-20- system) located over the right inferior gyrus of the $\mathrm{IPFC}$ was used as the dependent variable. Increases in this measure are thought to reflect decreased cortical activity, which should vary as a function of expectancy and correlate to control perception.

Hence, to investigate these relationships, two variables were calculated and included in a hierarchical mixed model. First, since no fixed sequence of reinforcer presence was provided, on a single trial level, inter- and intraindividual differences in presentation sequences emerge (between participants and between sessions within participants). Therefore a variable depicting the overall rate of positive outcomes to the point of the given trial was included in the model.

Furthermore, this effect should also alter immediate responses to the presence of a reinforcer in the context of its previous sequence of presentation. Hence, an estimator for the response to the presence of a reinforcer as well as an estimator for the effect of the overall presentation rate of the stimulus is reported. We anticipate a three-way interaction of overall reinforcement rate, litFUS, and the presence/absence of the reinforcer in a given trial. Furthermore, litFUS may increase control perception, by interfering with the processing of probabilities. Since the least amount of contingency/CP should result from reinforcer rates of 50\%, not only linear but quadratic functions are included, anticipating decreases in cortical activity at this rate but neither in rates above or below it.

\subsubsection{Midfrontal Theta mirrors conflict and effortful control.}

As mid-frontal theta is thought to reflect ACC activity, which is correlated to both effort and conflict, we hypothesize this measure to either correlate to worrying or effort (both self-reported). Furthermore, we hypothesize midfrontal theta to rely on the right IPFC processing, as it provides valuable information about the needed effort to attain a goal through its probabilistic processing. Thus, we also hypothesize mid-frontal theta to be directly modulated by the right IPFC's alpha density power.

\section{Methods}

\subsection{Sample.}

A total of 41 healthy volunteers were investigated after collecting written consent. Due to one participant taking part only in one session and issues with data collection in another case, only 39 data sets (consisting of two experimental sessions each) are analyzed. Participants were recruited via an online platform of the University of Wuerzburg, Germany, and postings on bulletin boards within the university. Of the remaining participants, 28 were female. The mean age was 23.9 (SD: 8.09). Participants were required to be at least 18 years old, not pregnant, right-handed, and without any previously diagnosed neurological or psychiatric diseases. All participants gave their consent to the publication of their data. The neuromodulation procedure was approved by the local ethics committee of the institute of psychology (reference: GZEK 2017-18) Further descriptive variables are presented in Table 1. 


\subsection{Material.}

The task was programmed in PsychoPy version 2.7 ([60]) and presented on a 24-inch screen (1920x1200 pixel). The rewarding stimulus was taken from emojipedia.org (Thumbs up sign, Apple IOS version 13.3). The thumbsup emoji was chosen to minimize confounding effects like interactions with the participant's gender (e.g. in response to faces) or other traits (like materialism in the context of monetary reward or loss).

\subsection{Procedure}

\subsubsection{Overall Setting.}

This experiment was part of a larger study that comprised three independent tasks (a reinforced go/no-go task and an internal shift task; [50]). Each participant visited the laboratory setting twice. The arrangement of tasks did not change between sessions, with this one to be presented last at each visit. It began approximately 20 minutes after the start of the experimental battery and approximately 40 minutes after litFUS administration. Before participants visited the EEG laboratory an online survey was completed, including several questionnaires that are relevant to the other two studies of the experimental set but are not reported here. The rewarding stimulus was solely used in this task but not in preceding tasks.

This study was conducted in a randomized- controlled double-blind cross-over design. Each volunteer received either sham or litFUS modulation at session one and the remaining at session two. The order of modulation was randomized across participants. Furthermore, neither experimenters nor participants knew whether a sham of litFUS was applied in a given session.

\subsubsection{Task.}

The task was largely designed following the task and instruction of Alloy, Abramson, and Viscusi ([5], p.1134). In the original study, the authors presented the judgementof-control problem, which was previously developed by the same group (Alloy and Abramson, [3]). Throughout 40 trials, participants were asked to either press a button or not. In $50 \%$ of the trials, a green light would appear, in the remaining $50 \%$, it would not. Afterward, participants were asked how much control they were able to exert on the green light. The author's results revealed greater control illusions in healthy as compared to depressed individuals, highlighting that depressed subjects showed a more accurate estimation of contingency.

The exact instruction is given on page 1134, in the paper of Alloy, Abramson, and Viscusi ([5]). This text was translated and used in the current study as well. In short, participants were asked to gain as much control as possible over the emergence of a positive reinforcer. In a time window of 3 seconds, they were asked to try to make the stimulus appear by pressing the space button as much as they wanted. They were also informed that the stimulus may sometimes appear, even though no button-press was made, and sometimes may stay away even though press(es) preceded. Contrary to the original study, participants were allowed to press as often as they wanted within one trial. A total of 40 trials were completed in each session. Also, just like in the original study, unbeknownst to the participants, the stimulus was presented completely at random leading to a Bernoulli-distributed succession of events. As a result, on a single trial level, the experience of participants with the reinforcer (e.g. by the number of trials including the reinforcer in the context of the overall number of trials) can be analyzed independently from immediate responses to its current presence of absence. However, the expected value of trials depicting the reinforcer was 20 . If shown, the stimulus remained present for 1 second.

After each session, participants rated the effort put into the task ("how much effort did you put into the task?"), the perceived control over the emergence of the stimulus("how much control did you gain over the emergence of the stimulus"?), their assumption on how well they performed in comparison to others ("how well did you do in comparison to others?") and their worrying about their performance ("how much did you worry about your performance?") on a 1100 visual analog scale (VAS). The ends of the VAS were described as "not at all" and "very much/good".

\section{4. litFUS Neuromodulation.}

Neuromodulation was applied at the F8 electrode position of the 10-20 EEG system. Its administration lasted for 120 seconds with a duty cycle of $0.5 \%$, a frequency of 500 $\mathrm{kHz}$, and a pulse repetition frequency of $40 \mathrm{~Hz}$ leading to an acoustic power of $199 \mathrm{~mW} / \mathrm{cm}^{2}$ and a mechanical index of 1.53. The ultrasound was emitted by a transducer, which connected to a gel pad that was directly placed on the F8 position of the scalp. Neuromodulation was operated by a manufactured device (Thync, Los Gatos, USA) and carried out by two experimenters at a time (one fixating the transducer and the gel pad to the participant's head and one starting the procedure on the device). While the two minutes of modulation/ sham were running, participants were asked not to move or talk to prevent distortions of the transducer relative to the targeted location. According to current computational models, litFUS neuromodulation with these parameters inhibits neural excitability ([61]).

Since ultrasonic waves are too high in frequency to be heard by humans, litFUS can easily be shammed. In this study, experimenters either pointed the transducer at the desired region of interest or in the opposite direction, away from the participant's scalp. However, litFUS was emitted, either way, keeping the preparation process for experimenters constant across litFUS- and sham-modulation. Nonetheless, depending on condition and session ( $\mathrm{t} 1$ vs. $\mathrm{t} 2$ ) experimenters were required to point the transducer in said directions without knowing which end of the transducer was the active one.

The same device was used before by Sanguinetti et al. [66], who used slightly altered parameters but targeted the same region of interest as this study does (for more details on 
the device and the method in general, please see Sanguinetti et al. [66]).

The wave propagation through the skull and neural tissue that results from the parameters used in this study was simulated using the SMART FUS (Surrogate model of attenuation and refraction in transcranial focused ultrasound) toolbox for MatLab ([18]). The result is shown in Figure 1

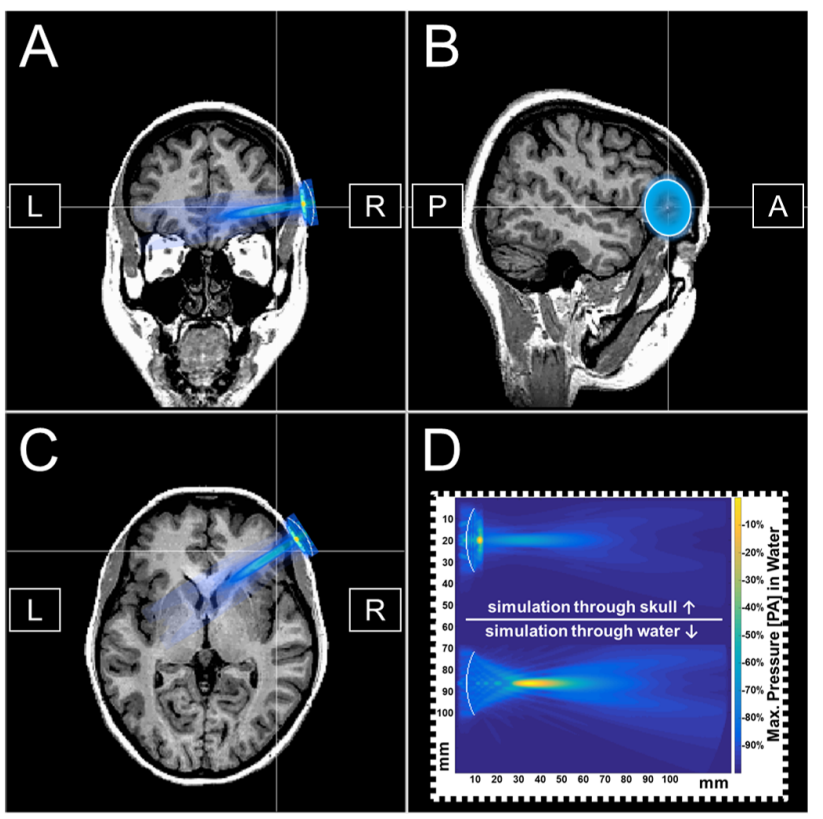

Figure 1: Estimated wave-propagation of ultrasound through skull and water. A) Coronal View. B) Sagittal View. C) Axial View. D) Simulation through the skull and water

\subsection{EEG recording and preprocessing.}

EEG recordings were collected via a 64- electrode montage and a brain vision recording system (Brain Products, Gilching, Germany). However, due to unforeseen technical issues, 13 participants were tested with a 32 electrode cap but an otherwise equal recording system. Bazanova and Vernon ([10]) report the cap montage to not influence alpha-related measures. Additionally, in all analyses depicted below, only those electrodes present in both sets were included. Electrodes were brought under $5 \mathrm{k} \Omega$. Online reference was $\mathrm{Cz}$, the ground was AFz.

The preprocessing was conducted via the Matlab (The MathWorks, Massachusetts, USA) extension EEGLAB ([28]) including plugins MARA ([78]), Adjust ([56]), SASICA ([23]), the CSD-toolbox ([43]; [44]) and the restingIAF toolbox ([25]). The procedure mostly followed Rodrigues' et al. ([64]) preprocessing pipeline: Electrodes were at first re-referenced to average. Afterward, channels were automatically rejected and interpolated based on their averaged z-score on the three dimensions of kurtosis, probability and spectrum. A z-score (calculated for the mean of one channel in comparison to all others) of more/less than \pm 3.29 (following suggestions by Tabachnik Fiedell [69] on outlier detection) qualified a channel for rejection. Epochs were extracted $(-300 \mathrm{~ms}$. to $1000 \mathrm{~ms}$.) and a $1 \mathrm{~Hz}$ high-pass filter was applied. Then, an ICA was conducted, and trials were automatically rejected by the same procedure as the channels had been. Following this, ICA weights and indices of rejected trials were saved. The preprocessing started once again and applied the saved parameters to the new set that was now missing the $1 \mathrm{~Hz}$ filter in its pipeline. Afterward, MARA was used to reject components based on Adjust and SASICA evaluations of the data. Finally, the data were CSDtransformed.

Following this, Morlet wavelets (fixed cycles of 3.5s, log-spaced) were used to extract the power of frequency bands that were identified individually for each participant by analyzing an eyes-closed resting condition that preceded the experimental test battery at each session via the restingIAF toolbox. Individual alpha frequency peaks (IAF) and bandwidth were determined by averaging calculated IAFs across a minimum of 17 channels (15 in 32 electrode setup) and a frequency range of 1-40 Hz. Bounds of the IAF search window were set to $7-13 \mathrm{~Hz}$ and a Savitzky-Golay filter of 11 bins was administered. The theta frequency band was set from the lower alpha bound to $2 \mathrm{~Hz}$ below the lower bound. All EEG measures were transformed via natural logarithms.

\subsection{Statistical analyses.}

All analyses were conducted via the R ( $\mathrm{R}$ Core Team, 2013) based package lme4 ([9]). The analyses follow the subsequent procedure: First, it is checked whether alpha at the F8 electrode position is influenced by the reinforcer rate and litFUS. Subsequently, the alpha value is used as a predictor for CP. Then, the influence of litFUS and alpha at F8 on MFT and associated self-ratings is investigated. Finally, exploratory analyses follow.

\subsubsection{Measures.}

To differentiate immediate right $1 \mathrm{PFC}$ reactions to the presence of the reinforcer and the influence of overall reinforcer presentation rates, two measures were included in the analyses: First, the presence and absence of the reinforcer in a given trial reflecting immediate reactions to a (un)expected emergence of a positive reinforcement cue. Second, a moving average was calculated and included. It was calculated by the number of reinforcements up to the previous trial, divided by the number of trials minus one. This equals the rate of reinforcer presence before the current trial, reflecting the current context of experience with the reinforcer. We chose to calculate the rate to the previous trial since this ensured independency of this effect from reinforcer presence in the current trial, which simplifies the interpretation of parameter estimates.

Furthermore, as level two variables, subjective control perception, effort, worrying, and internal attribution of one's performance were included as parameters. The latter was operationalized by the custom questions asking how well a participant thinks to have done in comparison to others. Higher scores are interpreted as an indicator of external attribution (e.g. "the task was rigged, this is uncontrollable for everyone, and thus I did well in comparison to others"). 
As a result, this variable needs to be included in the models as a control variable to negate confounding.

\subsubsection{General multilevel approach.}

The models reported below (section 3.2 and following) describe random intercept models (for each participant) as high intra-class correlations indicating their superiority over classical regression analyses. Furthermore, a random slope was included for the absolute number of reinforcer presentations up to the current trial. Metric predictors were grand mean centered, the two nominally scaled variables, litFUS and presence of the reinforcer, were simple coded to the references sham and presence.

The final models are summarized in Table 2 and 4, which also report and compare fit indices. Finally, the best fitting model was picked for parameter interpretation of fixed effects. Fixed effects were tested against a Bonferroni-Holm adjusted alpha.

\subsubsection{Role of the rIFG in control perception and reward processing.}

A set of models with an increasing number of parameters was calculated, including alpha density at F8 as the dependent variable. Table 2 and 3 give the complete set of model parameters. Subsequently, the best fitting model was chosen for further investigation of fixed effect parameters and tested for significance using the Satterwaite method. Furthermore, a separate mixed model, comprising the mean alpha response averaged across each respective session and the litFUS-term (levels: litFUS and sham) as independent variables as well as $\mathrm{CP}$ as the dependent variable, was fit. This model included only level-two variables and a random intercept for each participant.

\subsection{Mid-frontal theta mirrors conflict and effortful control.}

One set of mixed models was conducted including litFUS, worrying, and effort as variables. The complete set of variables in the final model is shown in Table 4 and 5.

\section{Results}

\subsection{Manipulation Check}

Table 1 depicts descriptives of the self-rated perception of control, internal attribution of performance, effort, and worrying alongside the individual alpha peak frequency. The results indicate $\mathrm{CP}$ to range in the lower- to middle range of the item's scale (1-101). Further, participants indicated that they exerted substantial effort and attributed their performance to their own (lack of) skill. However, no substantial difference between litFUS and sham seasons is present in these self-rate measures.

Regarding the neuromodulation procedure, time-frequency plots depicted in Figure 2 show increased alpha synchronization at around $10 \mathrm{~Hz}$ in response to the presence of the reinforcer during litFUS sessions. Furthermore, this effect is most prominent between 0 and $500 \mathrm{~ms}$. Thus, in the following analyses, EEG measures that were averaged across this time window and the respective individual alpha band for each participant's session were utilized.

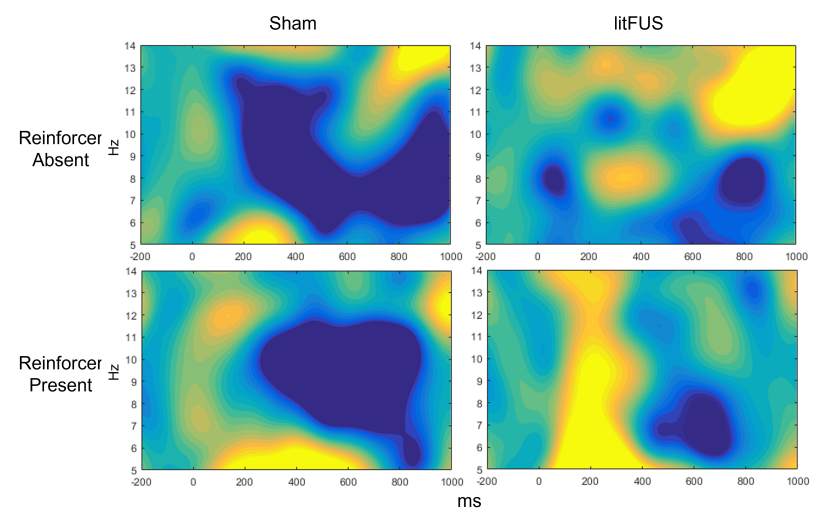

Figure 2: Time-frequency plot of electrode position F8.

\subsection{Role of the rIFG in control perception and reward processing.}

To clarify the role of the right lateral PFC in the aforementioned processes, a set of mixed effect regression models were calculated. Table 1 summarizes their model parameters. According to this, the best model is described by the inclusion of litFUS, presence of the reinforcing stimulus and the reinforcement rate. Furthermore, for each quadratic term, its linear pendant was also included. The results are reported in Table 2. Results indicate a significant influence of litFUS on alpha power at the F8 electrode position, which depends on the rate of reinforcer presence up to the previous trial. Figure 3a) further shows a weak quadratic relationship between reinforcer rate and alpha activity to be present in the sham condition, while a linear relationship is depicted in litFUS sessions. However, only the linear term remained significant after holm-correction.

Finally, to get a first impression of the IPFC's contribution to the perception of control (a level 2 variable), selfreport control perception was used as the dependent variable for a subsequent regression model. This model comprised the mean alpha response of each session averaged across (a) worse-than-chance trials (with a rate of <.4) and (b) betterthan-chance trials (with a rate of $>.6$ ). This type of analysis was chosen to avoid type-I error inflation by the prediction of level 2 variables with level 1 predictors such as singletrial reinforcer rates. The mean alpha response at worsethan chance trials was significantly able to predict control perception $(t(7.977)=-4.556, p=.002, \beta=-.72)$, while no such effect was present for alpha at better-than chance trials $(t(4.947)=1.766)$. This result indicates greater inactivity during worse-than-chance trials to predict lower control perception. However, in this analysis, only 29 participants were included since not every session comprised rates under $<.4$ and over $>$. 6 . 
Impact of the Right IPFC on Control Perception

$\begin{array}{llllll} & \text { IAF } & \text { Control Perception } & \text { Effort } & \text { Worrying } & \text { Internal Attribution of Performance } \\ \text { Sham } & 10.2(0.785) & 23.0(22.3) & 61.5(28.9) & 22.0(19.9) & 35.7(22.7) \\ \text { litFUS } & 9.98(0.673) & 22.5(21.2) & 62.5(27.4) & 20.1(21.6) & 36.1(23.1)\end{array}$

\section{Table 1}

Summary of self-ratings and IAF (individual alpha- peak frequency) for this studies sample including means and standard deviation

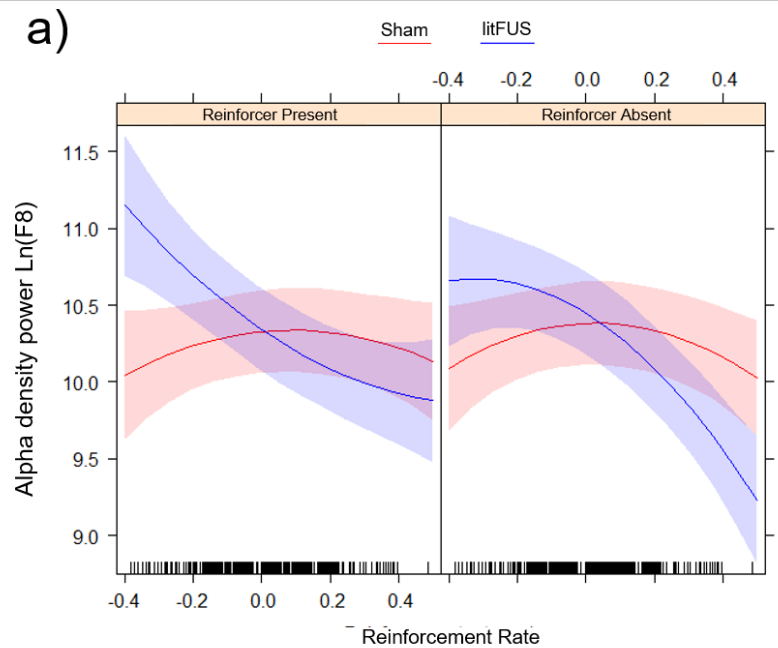

b)

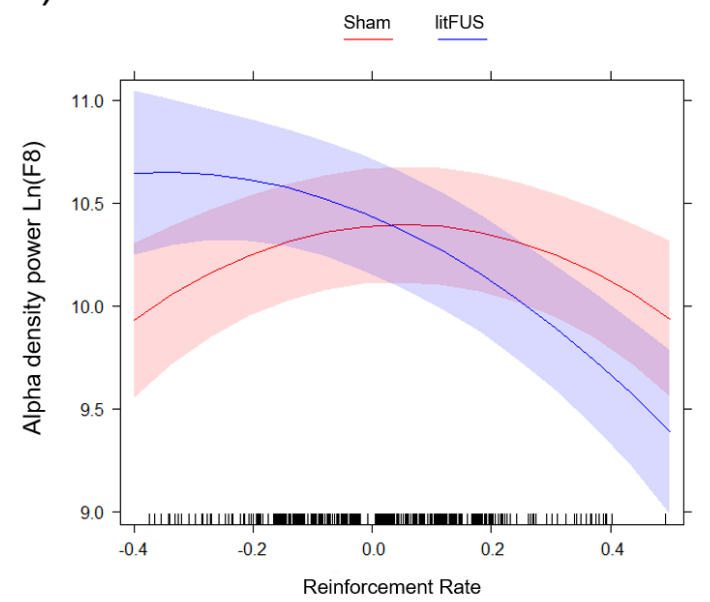

Figure 3: a) Estimated Relationship of alpha activity and reinforcer presence rates according to the model in Table 3. In the sham condition, a weak quadratic relationship is present, peaking at reinforcer rates of approximately .5. In litFUS sessions, a linear relationship seems to be present. Both effects seem largely irrespective of the reinforcer's presence in the given trial. b) Excluding the reinforcer presence from the analysis depicted in Table 3 and analyzing the alpha response to the rate of the current trial instead of the previous one, a more pronounced quadratic relationship emerges. Please note that the $x$-axis is grand mean-centered with zero equaling a rate of approximately .49 .

$\begin{array}{llllll} & \text { AIC } & \text { BIC } & \chi^{2} & \text { df } & p\left(\chi^{2}\right) \\ \text { RI } & 6868.2 & 6885.9 & & & \\ \text { RS } & 6865.9 & 6895.6 & 39.176 & 2 & .045^{*} \\ \text { Lvl1 } & 6817.3 & 6817.3 & 62.925 & 1 & <.001^{*} \\ \text { Lvl2 } & 6665.3 & 6730.5 & 46.681 & 5 & <.001^{*}\end{array}$

Table 2

Model comparisons of random intercept (RI) models with alpha density at $\mathrm{F} 8$ as the dependent variable. $\mathrm{AIC}=$ Alkaike information criterium, BIC= Bayesian information criterium, $\mathrm{df}=$ degrees of freedom. $\mathrm{RI}=$ random intercept model, $\mathrm{RS}=$ random intercept + slope model, Lvl1= RS + reinforcement rate and presence/absence, $\mathrm{Lvl} 2=\mathrm{Lvl} 1+$ litFUS

\subsection{Mid-frontal Theta mirrors conflict and effortful control.}

Again, a set of mixed models was compared to find the best fitting equation to describe the data. Afterward, fixed effects were interpreted and analyzed. The model comparison is summarized in Table 4

Mid-frontal theta activity was significantly negatively correlated to worrying. As seen in Table 4 and Figure 4, this relationship was nullified by litFUS compared to sham. Moreover, litFUS increased the relationship between effort and theta power density. Finally, alpha activity at F8 significantly predicted theta at $\mathrm{Fz}$ revealing a positive relationship, which indicates a correlation of midfrontal theta to less cortical activity at right $\mathrm{lpFC}$ sites.

\subsection{Additional, exploratory analyses}

In addition to F8, the final model shown in Table 3. was again investigated with its contralateral homologous site, F7, to check for hemispheric specificity of effects. Results show a somewhat different pattern, as none of the effects shown in Table 3 was significant. The results are shown in detail in Supplement 6.

Further, since we anticipated a quadratic relationship between reinforcer rate and alpha power, the mixed model depicted in Table 3 was refit without including the term for current reinforcer presence. By excluding this term we were now able to use the rate of the current trial instead of the previous one, as we did in Table 3 . The results indeed indicate a more pronounced quadratic relationship in the sham condition. Figure $4 \mathrm{~b}$ shows the estimated effect while detailed results can be reviewed in Supplement 7.

Finally, a random intercept model including litFUS and its interaction with IAF was carried out, revealing a highly significant interaction $(t(2777.80)=5.619, p($ holm $)<.001, \beta$ $=0.16$ ) that is illustrated in Figure 5 and shows that during sham sessions a higher individual alpha peak frequency is associated with less alpha power density. litFUS reverses this relationship with the point of intersection at approximately 
Impact of the Right IPFC on Control Perception

$\begin{array}{lrrrrrrr} & \text { Estimate } & \text { SE } & \text { df } & t & p & \text { holm } & \beta \\ \text { Rate:litFUS } & -1.752 & 0.349 & 2485.434 & -5.021 & <.001 & <.001 & -0.240 \\ \text { Rate }^{2} \text { :litFUS } & 2.431 & 1.090 & 2691.556 & 2.230 & .026 & .258 & 0.050 \\ \text { Rate }^{2} \text { :litFUS:Presence } & -2.941 & 1.462 & 2737.865 & -2.012 & .044 & .399 & -0.060 \\ \text { Rate }^{2} & -1.223 & 0.755 & 2576.198 & -1.619 & .106 & .844 & -0.030 \\ \text { Presence } & 0.055 & 0.049 & 2730.589 & 1.130 & .259 & 1 & 0.050 \\ \text { Rate } & 0.220 & 0.237 & 2534.440 & 0.927 & .354 & 1 & 0.030 \\ \text { litFUS:Presence } & 0.051 & 0.067 & 2722.271 & 0.772 & .440 & 1 & 0.040 \\ \text { Rate:litFUS:Presence } & 0.284 & 0.423 & 2733.499 & 0.672 & .502 & 1 & 0.040 \\ \text { Rate:Presence } & -0.127 & 0.292 & 2724.943 & -0.435 & .663 & 1 & -0.020 \\ \text { Rate } 2 \text { :Presence } & -0.383 & 0.985 & 2736.661 & -0.389 & .697 & 1 & -0.010 \\ \text { litFUS }_{\text {litFu }} & 0.014 & 0.048 & 2742.008 & 0.301 & .763 & 1 & 0.010\end{array}$

Table 3

Model parameters of the best model of Table $1 . \mathrm{SE}=$ standard error, $\mathrm{df}=$ degrees of freedom, holm=bonferroni-holm adjusted $p$-values
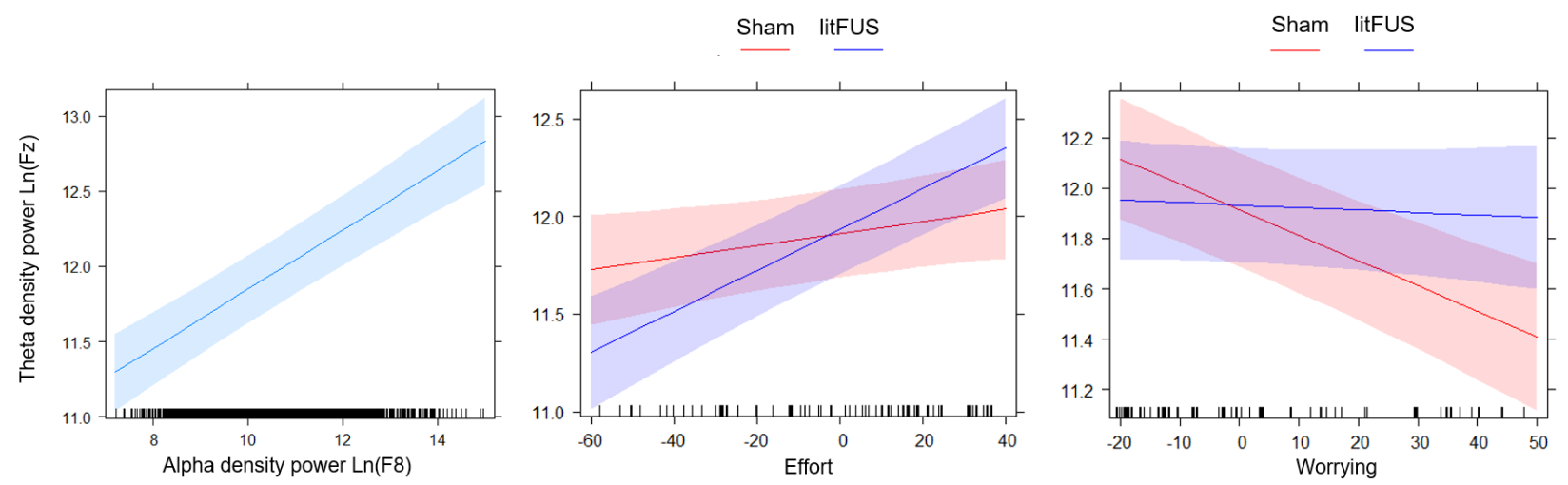

Figure 4: Effects of the model depicted in Table 5. While a strong relationship between alpha at F8 and theta at Fz exists, the correlation between effort and worrying is depending on litFUS. Please note that the $x$-axis are grand-mean centered

$\begin{array}{llllll} & \text { AIC } & \text { BIC } & \chi^{2} & \text { df } & p\left(\chi^{2}\right) \\ \text { RI } & 6705.9 & 6723.6 & & & \\ \text { RS } & 6700.9 & 6730.4 & 9.0209 & 2 & .011^{*} \\ \text { Lvl1 } & 6624.2 & 6665.5 & 80.7143 & 2 & <.001^{*} \\ \text { Lvl2 } & 6510.5 & 6575.4 & 121.6525 & 4 & <.001^{*}\end{array}$

Table 4

Model comparisons of random intercept (RI) models with theta density at $\mathrm{Fz}$ as dependent variable.AIC = Alkaike information criterion, $\mathrm{BIC}=$ Bayesian information criterion, $\mathrm{df}=$ degrees of freedom, $\mathrm{RI}=$ random intercept model, $\mathrm{RS}=$ random intercept + slope model, Lvl1=RS + Alpha(F8), Lvl2= Lvl1 + litFUS

$10 \mathrm{~Hz}$, which is in line with current theories regarding litFUS and the IAF.

\section{Discussion}

In this study, a multilevel modeling approach was used to dissociate the influence of current reinforcer presence and the overall probability of its presentation on the alpha response in the right $1 \mathrm{PFC}$, and its contribution to the illusion/perception of control. By using this type of hierarchical analysis and allowing cross-level interactions to predict single-trial variance, a model of context-dependent processing of current stimuli was introduced. Because reactivity of

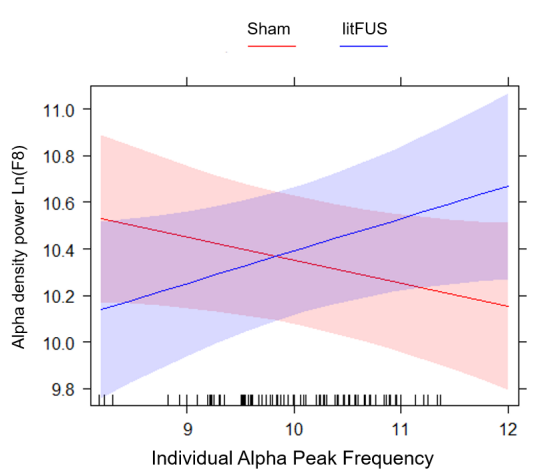

Figure 5: Interaction of litFUS and IAF in context of alpha activity at F8. While higher IAF predicts lower alpha levels across all trials during sham sessions, following litFUS this relationship is reversed. The estimated point of intersection is depicted at approximately $10 \mathrm{~Hz}$

the right $1 \mathrm{PFC}$ was manipulated by transcranial ultrasound neuromodulation within subjects, more direct attribution of results is possible.

Table 1 indicates that on average a significant illusion of control was induced. Table 1 also indicates that the belief to be able to control the reinforcer's presence (or the lack of it) 
Impact of the Right IPFC on Control Perception

$\begin{array}{llllllll} & \text { Estimate } & \mathrm{SE} & \mathrm{df} & t & p & h o l m & \beta \\ \text { Alpha(F8) } & 0.197 & 0.020 & 2755.600 & 10.046 & <.001^{*} & <.001^{*} & 0.19 \\ \text { litFUS*Effort } & 0.007 & 0.001 & 2756.110 & 5.637 & <.001^{*} & <.001^{*} & 0.17 \\ \text { litFUS*Worrying } & 0.009 & 0.002 & 2743.791 & -5.261 & <.001^{*} & <.001^{*} & 0.16 \\ \text { Worrying } & -0.010 & 0.002 & 2389.526 & -4.989 & <.001^{*} & <.001^{*} & -0.17 \\ \text { Effort } & 0.003 & 0.001 & 2235.945 & 2.139 & .032^{*} & .096 & 0.07 \\ \text { litFUS } & 0.019 & 0.030 & 2728.781 & 0.628 & .523 & .964 & 0.02\end{array}$

Table 5

Model parameters of the best model of Table 3. SE= standard error, $\mathrm{df}=$ degrees of freedom, Satt.=Satterhaite, holm=bonferroniholm adjusted p-values

is on average interpreted as the result of one's capability and (crucially) not as a property of the task, since participants posit themselves as doing worse than they would anticipate others to do. This notion is also supported by the fairly high self-reported effort put into the task. As a result, it can be argued that the overall objective of the task was met, even though no direct changes in these self-rate measures due to litFUS were present on average.

\subsection{Role of the right IPFC in control perception and reward processing}

The results depicted in Sections 3.2 and 3.4 (Figure 3b) show that in the sham condition, the right $1 \mathrm{PFC}$ reacted with increased alpha synchronization to rates near complete randomness (.5), which is in line with previous work by Fletcher et al. ([30]), who observed IPFC activity to be highest in learning phases and in response to surprising stimuli. Since alpha activity is widely regarded as an indicator of cortical inactivation, our results fit this notion: Increased activity is thought to reflect cognitive control exertion with the purpose of learning associations to gain predictability.

Following this idea, the quadratic relationship described in Sections 3.2 and 3.4 may thus reflect learning attempts that selectively take place in situations that signal some sort of noteworthy deviation from complete randomness (rates of .5). Additionally, it is worth noting that the reinforcerrate reported in this study included all previous trials. Thus, the resulting moving average cannot be not easily calculated by participants during task execution; hence participants may not have been able to explicitly perceive said rates. Therefore, the idea of a right IPFC-driven implicit learning process, taking into account the previous learning history to modulate the overall processing of current stimuli seems probable.

Interestingly, this quadratic effect seems to be significantly diminished when the reinforcer rate and current presence of the reinforcer are divided into two separate factors (see the model in Table 3, which used the reinforcer rate of the previous trial, and the current reinforcer presence to investigate independent effects of current and previous experiences). Conversely, it is more significant when the current reinforcer presentation is mixed into the presentation rate. This suggests that the quadratic relationship between reinforcer rate and alpha power occurs primarily when current experience with the stimulus is included in the calculation. This, in turn, indicates that the processing of probabilistic information takes place mainly against the background of current updates of the respective appearance rate. Furthermore, the right 1PFC's alpha response did not seem to react to the presence of the reinforcer in the given trial aside from its contribution to this probabilistic processing. Additionally, this seems to be true without taking the person's action into account, as this variable was neither included in the model nor indicative of the outcome at all (since this is a task with the completely randomized presentation of reinforcers). As a result, this indicates a general reaction to positive stimuli rather than contingent outcomes.

Also, this study was able to show that in particular, the alpha response to worse-than-chance trials predicted overall control perception. In line with the findings of Lorenz et al. ([51], see section 1.1.), greater rIFG activity predicted increased control illusion. However, concerning the specificity of worse-than-chance trials, this effect may stem from the design of this task specifically since no real control could be attained. As a result, being able to interpret the above-chance absence of the reinforcer as a significant event that holds information doubles the content to possibly learn from. Greater control perception may follow. Thus, even though the current data provide first hints towards a special contribution of worse-than-expected learning in the right IPFC, further research is needed to support this idea in other experimental tasks. This is further especially true since only a fraction of the complete sample could be included in the analysis leading to this notion.

Finally, this study points out that the right $1 \mathrm{PFC}$ has a (possibly) unique ability to model stochastic data as none of the F8-related effects could be replicated at F7.

\subsection{Mid-frontal Theta mirrors conflict and effortful control.}

The second objective of this study was to investigate the use of litFUS to alter ACC-related cognitive reactions to the perception of low controllability.

Table 5 shows that in the sham condition theta activity at the mid-frontal electrode $\mathrm{Fz}$ is negatively correlated to worrying, which may imply that increased cognitive control, which may indicate learning attempts or conflict resolution attempts (indexed by MFT) to go along with decreases in worrying.

However, this result is not in line with the current literature since greater theta activity is usually connected to anxious responses (e.g. [59]). As a result, one may argue 
that worrying, a cognitive facet of anxiety, should also be positively connected to MFT responses. This effect should be most prominent in situations of uncertainty about threat ([Cavanagh.2012]). However, this task may not have been able to induce such anxious responses, since no relevant threat was anticipated in response to performance deficits (e.g. in contrast to the experience in helplessness tasks). Furthermore, theta activity was also positively correlated to alpha power at the F8 electrode position, which is unusual given that theta oscillations are usually interpreted to reflect cortical activity, while alpha oscillations indicate cortical inhibition. In sum, these results imply that theta activity may reflect other cognitive/emotional states in such a control illusion task than it does in tasks that rely on cognitive control over dominant behavior (e.g. in go/no-go, flanker, or Stroop tasks) or behavioral adaptation in the context of threat.

\section{3. litFUS and the individual alpha peak frequency}

This study was built on a within-person comparison of effects between two experimental sessions, once following litFUS and once following a sham modulation. Before the background of the NICE model, this comparison should depend in part on the activity of receptors that fire at a rate of approximately $10 \mathrm{~Hz}$, thus increasing oscillations in the range of the alpha frequency. In this regard, this study provides the first empirical evidence for the applicability of the NICE model ([61]) by showing an individual alpha peak frequency dependent effect of litFUS, which is in line with evidence indicating this IAF to rely on interindividual differences in the same receptor type that is implicated in the NICE model. Interestingly, such IAF-dependent effects of neuromodulation have been reported in other techniques, such as TMS as well (e.g., [26]). The IAF is thought to reflect the preferred oscillation frequency by specific neural networks. As such, especially the theta band has been shown to convey functioning and network connectivity of the default mode network (DMN; e.g., [33]), which is correlated to worrying and rumination but also cognitive control (e.g., [24]; [40]). Thus, differential effects of litFUS on individuals that show different IAFs, are well in line with findings from other methods and may also imply that the effects shown above are to some extent connected to altered activity in the DMN. This, in turn, would also be in line with the correlation between ACC and IPFC activity shown above. Furthermore, alterations in functional connectivity of the DMN have already been shown in litFUS neuromodulation of the same region ([66]).

\subsection{1. litFUS' influence on alpha power and control perception}

As discussed in 3.2 litFUS conveyed inhibition of the right IPFC moves the probabilistic processing effect peak to lower reinforcement rates by forming a linear rather than quadratic relationship between reinforcer rate and alpha activity.
However, no difference in control perception was present between the sessions. Even though alpha responses in worsethan-chance trials predicted control perception and litFUS was able to modulate the right lPFC's response in such trials, the effect does not seem great enough to show a mediationlike effect of litFUS on CP, which may hint towards other contributing areas or factors that were not taken into account or to power issues regarding the sample size, modulation intensity, or experimental procedure. Thus, this study provides preliminary evidence for the relationship between the right IPFC and control perception but failed to provide direct insight into the causal influence of the area, since no direct effect of litFUS on CP was present.

\subsection{2. litFUS' influence on MFT-related results}

litFUS was found to alter the relationships discussed in Section 3.3. within the sample, significantly. First, litFUS was able to decouple ACC-driven theta from worrying. Fittingly, a recently published study shows that litFUSmediated inhibition of the rIFG decreases worrying and anxiety. According to Sanguinetti et al. ([66]) rIFG inhibition via litFUS was able to decrease the functional connectivity of networks related to worrying and mood.

Furthermore, litFUS introduced a significant correlation between self-reported effort and theta density power at Fz. As mentioned above, the right IPFC hast strong inhibitory projections to subcortical structures, including the VS. The VS on the other hand carries out part of the reward estimation and uses dopaminergic projections to inform the ACC about the current rewarding status, as well as "better" and "worse than expected" values. Subsequently, the ACC then chooses tasks, behavior, and goals on this basis and exerts the minimum amount of control over other cortical areas that are needed to come closer to a goal (see section 1.2.). The ACC does this via frontal theta synchronization. As a result, disinhibition of the VS by litFUS modulation of the rIFG may lead to amplified reward valuation and thus increase the relationship between ACC-emitted theta and goal approach (effort) while diminishing its correlation to conflict-related cognition (e.g. not being able to find an appropriate behavior) including worrying.

\subsection{Summary}

In summary, these data provide overall evidence for the involvement of the right $\mathrm{PFC}$ in the processing of control perception by contributing information based on stochastic estimation. Since control perception is thought to build upon action-outcome contingency, the attribution of outcomes to a certain behavior is crucial. However, if this attribution is complicated by factors of uncertainty (e.g. in situations of only intermittently occurring action-outcome contingency), estimation of conditional probabilities provides an important context. In the current study, the right IPFC's inactivity is greatest in response to patterns that depict randomness, which is interpreted as an indicator for decreased associative learning. Thus, we conclude the rIFG of the IPFC to integrate probabilistic context information with current stimulus processing, thereby modulating the experience of contingency. 
Finally, this was found to predict control illusion and ACCrelated MFT. These effects were unique to the right hemisphere and were not present at the left IPFC.

In addition, inhibition of the right $\mathrm{PFC}$ induced decoupling from worrying and increased its correlation to the self-reported effort. As a result, one might argue that modulation of the right IPFC also modulated activity in a broader network, including ACC functioning.

With regard to control perception being one of the most discussed influences in the emergence of helplessness, this study indicates that neuromodulation of the PFC may contribute to a state that allows patients to experience positive events as a consequence of their action/cognition/emotion/state more often. Thus, especially neuromodulation before therapy sessions, meditation, or similar situations may benefit psychiatric patients and those at risk.

Nonetheless, given the current data, further research is needed to replicate these results and enrich them with multi-level analyses-based findings. On that note, especially level 1 (trial-by-trial or block-by-block) control-perception measures of some kind would greatly benefit the discussion and significantly improve statistical power. Furthermore, additional (e.g., fMRI) data on the subject of probabilistic learning in the right IPFC will be needed to clarify the role of alpha activity in this study.

\section{References}

[1] I. Ajzen. "Perceived Behavioral Control, Self-Efficacy, Locus of Control, and the Theory of Planned Behavior". In: Journal of Applied Social Psychology 32 (2002), pp. 1-20. ISSN: 1559-1816.

[2] William H. Alexander and Joshua W. Brown. "Medial prefrontal cortex as an action-outcome predictor". In: Nature neuroscience 14.10 (2011), pp. 1338-1344. DOI: 10.1038/nn. 2921.

[3] Lauren B. Alloy and Lyn Y. Abramson. "Judgment of contingency in depressed and nondepressed students: Sadder but wiser?" In: Journal of experimental psychology: general 108.4 (1979), p. 441. ISSN: 1939-2222.

[4] Lauren B. Alloy and Lyn Y. Abramson. "Learned helplessness, depression, and the illusion of control". In: Journal of personality and social psychology 42.6 (1982), p. 1114. ISSN: 1939-1315.

[5] Lauren B. Alloy, Lyn Y. Abramson, and Donald Viscusi. "Induced mood and the illusion of control". In: Journal of personality and social psychology 41.6 (1981), p. 1129. ISSN: 1939-1315.

[6] Lauren B. Alloy and Caroline M. Clements. "Illusion of control: Invulnerability to negative affect and depressive symptoms after laboratory and natural stressors". In: Journal of abnormal Psychology 101.2 (1992), p. 234. ISSN: 1939-1846.

[7] Sarah Alsawy et al. "Science and practice of transdiagnostic CBT: a Perceptual Control Theory (PCT) approach". In: International Journal of Cognitive Therapy 7.4 (2014), pp. 334-359. ISSN: 19371209.

[8] Ilya Baryshnikov et al. "State and trait hopelessness in a prospective five-year study of patients with depressive disorders". In: Journal of affective disorders 239 (2018), pp. 107-114. ISSN: 0165-0327.

[9] Douglas Bates et al. "Parsimonious mixed models". In: arXiv preprint arXiv:1506.04967 (2015).

[10] O. M. Bazanova and D. Vernon. "Interpreting EEG alpha activity". In: Neuroscience \& Biobehavioral Reviews 44 (2014), pp. 94-110. ISSN: 0149-7634.
[11] Fernando Blanco, Helena Matute, and Miguel A. Vadillo. "Making the uncontrollable seem controllable: The role of action in the illusion of control". In: Quarterly Journal of Experimental Psychology 64.7 (2011), pp. 1290-1304. ISSN: 1747-0218.

[12] Anil Bollimunta et al. "Neuronal mechanisms and attentional modulation of corticothalamic alpha oscillations". In: Journal of Neuroscience 31.13 (2011), pp. 4935-4943. ISSN: 0270-6474.

[13] Jeffrey J. Borckardt et al. "Fast left prefrontal rTMS acutely suppresses analgesic effects of perceived controllability on the emotional component of pain experience". In: PAIN@ 152.1 (2011), pp. 182-187. ISSN: 0304-3959.

[14] Matthew M. Botvinick et al. "Conflict monitoring and cognitive control”. In: Psychological review 108.3 (2001), p. 624. ISSN: 19391471.

[15] Damian P. Bright, M. Isabel Aller, and Stephen G. Brickley. "Synaptic release generates a tonic GABAA receptor-mediated conductance that modulates burst precision in thalamic relay neurons". In: Journal of Neuroscience 27.10 (2007), pp. 2560-2569. ISSN: 02706474.

[16] Jonathon D. Brown and Judith M. Siegel. "Attributions for negative life events and depression: The role of perceived control". In: Journal of personality and social psychology 54.2 (1988), p. 316. ISSN: 1939-1315.

[17] Jerry M. Burger and Robert M. Arkin. "Prediction, control, and learned helplessness". In: Journal of Personality and Social Psychology 38.3 (1980), pp. 482-491. ISSN: 0022-3514. DOI: 10.1037/ $0022-3514.38 .3 .482$.

[18] Joshua Cain. SMART FUS: Surrogate model of attenuation and refraction in transcranial focused ultrasound. 2022. DOI: 10.5281/ zenodo. 5811121.

[19] James F. Cavanagh, Michael X. Cohen, and John J. B. Allen. "Prelude to and resolution of an error: EEG phase synchrony reveals cognitive control dynamics during action monitoring". In: Journal of Neuroscience 29.1 (2009), pp. 98-105. ISSN: 0270-6474.

[20] James F. Cavanagh and Michael J. Frank. "Frontal theta as a mechanism for cognitive control". In: Trends in cognitive sciences 18.8 (2014), pp. 414-421. ISSN: 1364-6613.

[21] James F. Cavanagh and Alexander J. Shackman. "Frontal midline theta reflects anxiety and cognitive control: meta-analytic evidence". In: Journal of physiology-Paris 109.1-3 (2015), pp. 3-15. ISSN: 0928-4257.

[22] Valérian Chambon, James W. Moore, and Patrick Haggard. "TMS stimulation over the inferior parietal cortex disrupts prospective sense of agency". In: Brain Structure and Function 220.6 (2015), pp. 3627-3639. ISSN: 1863-2661.

[23] Maximilien Chaumon, Dorothy V. M. Bishop, and Niko A. Busch. "A practical guide to the selection of independent components of the electroencephalogram for artifact correction". In: Journal of neuroscience methods 250 (2015), pp. 47-63. ISSN: 0165-0270.

[24] Rebecca E. Cooney et al. "Neural correlates of rumination in depression". In: Cognitive, affective \& behavioral neuroscience 10.4 (2010), pp. 470-478. DOI: 10.3758/CABN. 10.4.470.

[25] Andrew W. Corcoran et al. "Toward a reliable, automated method of individual alpha frequency (IAF) quantification”. In: Psychophysiology 55.7 (2018), e13064. ISSN: 0048-5772.

[26] Juliana Corlier et al. "The relationship between individual alpha peak frequency and clinical outcome with repetitive Transcranial Magnetic Stimulation (rTMS) treatment of Major Depressive Disorder (MDD)". In: Brain stimulation 12.6 (2019), pp. 1572-1578. ISSN: 1935-861X. DOI: 10.1016/j.brs.2019.07.018.

[27] James Danckert et al. "Right hemisphere brain damage impairs strategy updating”. In: Cerebral cortex 22.12 (2012), pp. 2745-2760. ISSN: 1460-2199. 
[28] Arnaud Delorme and Scott Makeig. "EEGLAB: an open source toolbox for analysis of single-trial EEG dynamics including independent component analysis". In: Journal of neuroscience methods 134.1 (2004), pp. 9-21. ISSN: 0165-0270.

[29] Alex Filipowicz, Britt Anderson, and James Danckert. "Adapting to change: The role of the right hemisphere in mental model building and updating". In: Canadian Journal of Experimental Psychology/Revue canadienne de psychologie expérimentale 70.3 (2016), p. 201. ISSN: 1878-7290.

[30] Paul C. Fletcher et al. "Responses of human frontal cortex to surprising events are predicted by formal associative learning theory". In: Nature neuroscience 4.10 (2001), pp. 1043-1048. ISSN: 1546-1726.

[31] Matthew W. Gallagher, Kristin Naragon-Gainey, and Timothy A. Brown. "Perceived control is a transdiagnostic predictor of cognitive-behavior therapy outcome for anxiety disorders". In: Cognitive therapy and research 38.1 (2014), pp. 10-22. ISSN: 01475916.

[32] Sanford Golin, Francis Terrell, and Barbara Johnson. "Depression and the illusion of control". In: Journal of abnormal Psychology 86.4 (1977), p. 440. ISSN: 1939-1846.

[33] Carl D. Hacker et al. "Frequency-specific electrophysiologic correlates of resting state fMRI networks". In: Neuroimage 149 (2017), pp. 446-457. ISSN: 1053-8119. DOI: 10.1016/j . neuroimage. 2017.01. 054.

[34] Stuart Hameroff et al. "Transcranial ultrasound (TUS) effects on mental states: a pilot study". In: Brain stimulation 6.3 (2013), pp. 409-415. ISSN: 1935-861X.

[35] David Hecht, Vincent Walsh, and Michal Lavidor. "Transcranial direct current stimulation facilitates decision making in a probabilistic guessing task". In: Journal of Neuroscience 30.12 (2010), pp. 4241-4245. ISSN: 0270-6474.

[36] Sanne M. Hogendoorn et al. "Mediators of cognitive behavioral therapy for anxiety-disordered children and adolescents: Cognition, perceived control, and coping". In: Journal of Clinical Child \& Adolescent Psychology 43.3 (2014), pp. 486-500. ISSN: 1537-4416.

[37] Clay B. Holroyd and Akina Umemoto. "The research domain criteria framework: The case for anterior cingulate cortex". In: Neuroscience \& Biobehavioral Reviews 71 (2016), pp. 418-443. ISSN: 0149-7634.

[38] Stuart Hughes et al. "Thalamic gap junctions control local neuronal synchrony and influence macroscopic oscillation amplitude during EEG alpha rhythms". In: Frontiers in psychology 2 (2011), p. 193. ISSN: 1664-1078.

[39] Stuart W. Hughes and Vincenzo Crunelli. "Thalamic mechanisms of EEG alpha rhythms and their pathological implications". In: The Neuroscientist 11.4 (2005), pp. 357-372. ISSN: 1073-8584.

[40] Rachel H. Jacobs et al. "Increased coupling of intrinsic networks in remitted depressed youth predicts rumination and cognitive control". In: PloS one 9.8 (2014), e 104366. DOI: 10.1371/journal . pone. 0104366.

[41] Karolina Janacsek et al. "Right hemisphere advantage in statistical learning: evidence from a probabilistic sequence learning task". In: Brain stimulation 8.2 (2015), pp. 277-282. ISSN: 1935-861X.

[42] Kevin Johnston et al. "Top-down control-signal dynamics in anterior cingulate and prefrontal cortex neurons following task switching". In: Neuron 53.3 (2007), pp. 453-462. ISSN: 0896-6273. DOI: 10. 1016/j. neuron. 2006.12.023.

[43] Jürgen Kayser and Craig E. Tenke. "Principal components analysis of Laplacian waveforms as a generic method for identifying ERP generator patterns: I. Evaluation with auditory oddball tasks". In: Clinical neurophysiology 117.2 (2006), pp. 348-368. ISSN: 13882457.
[44] Jürgen Kayser and Craig E. Tenke. "Principal components analysis of Laplacian waveforms as a generic method for identifying ERP generator patterns: II. Adequacy of low-density estimates". In: Clinical neurophysiology 117.2 (2006), pp. 369-380. ISSN: 1388-2457.

[45] Steven W. Kennerley et al. "Optimal decision making and the anterior cingulate cortex". In: Nature neuroscience 9.7 (2006), pp. 940-947. ISSN: 1546-1726.

[46] Hyungmin Kim et al. "Suppression of EEG visual-evoked potentials in rats via neuromodulatory focused ultrasound". In: Neuroreport 26.4 (2015), p. 211.

[47] Marie K. Krug and Cameron S. Carter. "Proactive and reactive control during emotional interference and its relationship to trait anxiety". In: Brain research 1481 (2012), pp. 13-36. DOI: 10.1016/ j.brainres.2012.08.045.

[48] Julie Ledrich and Kamel Gana. "Relationship between attributional style, perceived control, self-esteem, and depressive mood in a nonclinical sample: A structural equation-modelling approach". In: Psychology and Psychotherapy: Theory, Research and Practice 86.4 (2013), pp. 413-430. ISSN: 1476-0835.

[49] Wynn Legon et al. "Neuromodulation with single-element transcranial focused ultrasound in human thalamus". In: Human brain mapping 39.5 (2018), pp. 1995-2006. ISSN: 1065-9471.

[50] Evi de Lissnyder, Ernst H. W. Koster, and Rudi de Raedt. "Emotional Interference in Working Memory is Related to Rumination". In: Cognitive therapy and research 36.4 (2012), pp. 348-357. ISSN: 0147-5916. DOI: 10.1007/s10608-011-9352-4.

[51] Robert C. Lorenz et al. "Subjective illusion of control modulates striatal reward anticipation in adolescence". In: Neuroimage 117 (2015), pp. 250-257. ISSN: 1053-8119.

[52] Steven F. Maier and Martin E. Seligman. "Learned helplessness: theory and evidence". In: Journal of experimental psychology: general 105.1 (1976), p. 3. ISSN: 1939-2222.

[53] Steven F. Maier and Martin E. P. Seligman. "Learned helplessness at fifty: Insights from neuroscience". In: Psychological review 123.4 (2016), p. 349. ISSN: 1939-1471.

[54] Helena Matute. "Illusion of control: Detecting response-outcome independence in analytic but not in naturalistic conditions". In: Psychological Science 7.5 (1996), pp. 289-293. ISSN: 0956-7976.

[55] Michael B. Miller and Monica Valsangkar-Smyth. "Probability matching in the right hemisphere". In: Brain and cognition 57.2 (2005), pp. 165-167. ISSN: 0278-2626.

[56] Andrea Mognon et al. "ADJUST: An automatic EEG artifact detector based on the joint use of spatial and temporal features". In: Psychophysiology 48.2 (2011), pp. 229-240. ISSN: 0048-5772.

[57] Yael Niv. "Reinforcement learning in the brain". In: Journal of Mathematical Psychology 53.3 (2009), pp. 139-154. ISSN: 00222496.

[58] A. O'Dhaniel et al. "Infrequent, task-irrelevant monetary gains and losses engage dorsolateral and ventrolateral prefrontal cortex". In: Brain research 1395 (2011), pp. 53-61. ISSN: 0006-8993.

[59] Roman Osinsky, Christian Karl, and Johannes Hewig. "Dispositional Anxiety and Frontal-Midline Theta: On the Modulatory Influence of Sex and Situational Threat". In: Journal of personality 85.3 (2017), pp. 300-312. DOI: 10.1111/jopy. 12241.

[60] Jonathan W. Peirce. "Generating stimuli for neuroscience using PsychoPy". In: Frontiers in neuroinformatics 2 (2009), p. 10. ISSN: 1662-5196.

[61] Michael Plaksin, Eitan Kimmel, and Shy Shoham. "Cell-typeselective effects of intramembrane cavitation as a unifying theoretical framework for ultrasonic neuromodulation". In: Eneuro 3.3 (2016). 
[62] Paul K. Presson and Victor A. Benassi. "Illusion of control: A metaanalytic review". In: Journal of social behavior and personality 11.3 (1996), p. 493. ISSN: 0886-1641.

[63] Samantha J. Reznik et al. "A double-blind pilot study of transcranial ultrasound (TUS) as a five-day intervention: TUS mitigates worry among depressed participants". In: Neurology, Psychiatry and Brain Research 37 (2020), pp. 60-66. ISSN: 0941-9500.

[64] Johannes Rodrigues et al. "EPOS: EEG Processing Open-Source Scripts". In: Frontiers in neuroscience 15 (2021), p. 660449. ISSN: 1662-4548. DOI: 10.3389/fnins. 2021.660449.

[65] Matthew E. Roser et al. "Right hemisphere dominance in visual statistical learning". In: Journal of cognitive neuroscience 23.5 (2011), pp. 1088-1099. ISSN: 0898-929X.

[66] Joseph L. Sanguinetti et al. "Transcranial focused ultrasound to the right prefrontal cortex improves mood and alters functional connectivity in humans". In: Frontiers in human neuroscience 14 (2020), p. 52. ISSN: 1662-5161.

[67] Rebecca Levin Silton et al. "The time course of activity in dorsolateral prefrontal cortex and anterior cingulate cortex during top-down attentional control". In: NeuroImage 50.3 (2010), pp. 1292-1302. DOI: $10.1016 / j$. neuroimage. 2009.12 .061 .

[68] Elisabeth Stöttinger et al. "Statistical and perceptual updating: correlated impairments in right brain injury". In: Experimental brain research 232.6 (2014), pp. 1971-1987. ISSN: 0014-4819.

[69] Barbara G. Tabachnick, Linda S. Fidell, and Jodie B. Ullman. Using multivariate statistics. Pearson Boston, MA, 2007.

[70] Yusuf Tufail et al. "Transcranial pulsed ultrasound stimulates intact brain circuits”. In: Neuron 66.5 (2010), pp. 681-694. ISSN: 08966273.

[71] Danielle C. Turner et al. "The role of the lateral frontal cortex in causal associative learning: exploring preventative and superlearning". In: Cerebral cortex (New York, N.Y. : 1991) 14.8 (2004), pp. 872-880. ISSN: 1047-3211. DOI: 10.1093/cercor/bhh046.

[72] William J. Tyler. "The mechanobiology of brain function". In: Nature Reviews Neuroscience 13.12 (2012), pp. 867-878. ISSN: 1471-0048.

[73] Michiel van Elk, Bastiaan T. Rutjens, and Joop van der Pligt. "The development of the illusion of control and sense of agency in 7-to12-year old children and adults". In: Cognition 145 (2015), pp. 1-12. ISSN: 0010-0277.

[74] Marie-Anne Vanderhasselt et al. "The influence of rTMS over the right dorsolateral prefrontal cortex on top-down attentional processes". In: Brain research 1137.1 (2007), pp. 111-116. DOI: 10. 1016/j. brainres. 2006.12.050.

[75] Sven Vanneste and Dirk de Ridder. "The involvement of the left ventrolateral prefrontal cortex in tinnitus: a TMS study". In: Experimental brain research 221.3 (2012), pp. 345-350. ISSN: 0014-4819.

[76] Kenneth A. Wallston et al. "Perceived control and health". In: Current Psychology 6.1 (1987), pp. 5-25. ISSN: 1936-4733.

[77] Katja Wiech et al. "Anterolateral prefrontal cortex mediates the analgesic effect of expected and perceived control over pain". In: The Journal of neuroscience : the official journal of the Society for Neuroscience 26.44 (2006), pp. 11501-11509. DOI: 10.1523 / JNEUROSCI . 2568-06.2006.

[78] Irene Winkler, Stefan Haufe, and Michael Tangermann. "Automatic classification of artifactual ICA-components for artifact removal in EEG signals". In: Behavioral and Brain Functions 7.1 (2011), pp. 1-15. ISSN: 1744-9081.

[79] George Wolford, Michael B. Miller, and Michael Gazzaniga. "The left hemisphere's role in hypothesis formation". In: Journal of Neuroscience 20.6 (2000), RC64-RC64. ISSN: 0270-6474.
[80] Seung-Schik Yoo et al. "Transcranial focused ultrasound to the thalamus alters anesthesia time in rats". In: Neuroreport 22.15 (2011), p. 783. 
Impact of the Right IPFC on Control Perception

5. Appendix

Rate

Rate $^{2}$

Presence

litFUS

Rate:Presence

Rate $^{2}$ :Presence

Rate:litFUS

Rate $^{2}$ :litFUS

litFUS:Presence

Rate:litFUS:Presence

Rate $^{2}:$ litFUS:Presence
Estimate

0.360

$-1.514$

0.012

$-0.074$

$-0.142$

1.082

$-0.599$

2.094

0.087

0.380

$-3.484$
SE

0.229

0.731

0.048

0.046

0.283

0.955

0.337

1.055

0.065

0.410

1.416 df

2493.150

$t$

1.570

$p$

0.116

0.039

0.795

0.110

0.616

0.257

0.076

0.047

0.181

0.354

0.014

$\beta$

0.05

$-0.03$

0.01

$-0.07$

$-0.02$

0.02

$-0.09$

0.05

0.08

0.05

\subsection{5}

$-2.459$ $-0.08$

Table 6

Estimated effects of the same model depicted in Table 3 concerning the F7 electrode position instead of F8

\begin{tabular}{|c|c|c|c|c|c|c|}
\hline & Estimate & SE & $d f$ & $t$ & $p$ & $\beta$ \\
\hline Rate & 2.54 & 0.70 & 2754.05 & 3.65 & 0.00 & 0.03 \\
\hline $\mathrm{te}^{2}$ & -2.27 & 0.59 & 2752.39 & -3.85 & 0.00 & -0.04 \\
\hline & 0.92 & 0.29 & 2757.21 & 3.14 & 0.00 & 0.04 \\
\hline & -2.00 & 1.04 & 2753.67 & -1.93 & 0.05 & -0.18 \\
\hline $\mathrm{te}^{2}:$ litFUS & 0.54 & 0.89 & 2750.66 & 0.61 & 0.55 & 0.01 \\
\hline
\end{tabular}

Table 7

Results of the model shown in Table 3, excluding the term for current reinforcer presence and exchanging rate calculated for the previous trial with the current rate 